\title{
Nutritional assessment in esophageal fast-track surgery: comparisons of 4 objective malnutrition screening tools
}

\author{
Xiong-Huai Hua ${ }^{1}$, Ke-Feng Shi ${ }^{1}$, Yong-Kui Yu ${ }^{2}$, Hao-Miao $\mathrm{Li}^{2}$, Fei Ma ${ }^{3}$, Hai-Bo Sun ${ }^{2}$, Ru-Lin Qian ${ }^{1}$, \\ Yin $\mathrm{Li}^{4}$
}

${ }^{1}$ Department of Thoracic Surgery, Henan Chest Hospital, Zhengzhou, China; ${ }^{2}$ Department of Thoracic Surgery, The Affiliated Cancer Hospital of Zhengzhou University, Henan Cancer Hospital, Zhengzhou, China; ${ }^{3}$ Department of Gastrointestinal Surgery, The Affiliated Cancer Hospital of Zhengzhou University, Henan Cancer Hospital, Zhengzhou, China; ${ }^{4}$ Department of Thoracic Surgical Oncology, National Cancer Center/Cancer Hospital, Chinese Academy of Medical Sciences and Peking Union Medical College, Beijing, China

Contributions: (I) Conception and design: XH Hua, YK Yu, Y Li; (II) Administrative support: XH Hua, RL Qian, Y Li; (III) Provision of study materials or patients: XH Hua, YK Yu, HM Li, HB Sun; (IV) Collection and assembly of data: XH Hua, YK Yu, F Ma, KF Shi, HB Sun; (V) Data analysis and interpretation: XH Hua, KF Shi, HM Li, F Ma, HB Sun; (VI) Manuscript writing: All authors; (VII) Final approval of manuscript: All authors.

Correspondence to: Yin Li, MD, PhD. Department of Thoracic Surgical Oncology, National Cancer Center/Cancer Hospital, Chinese Academy of Medical Sciences and Peking Union Medical College, Beijing 100021, China. Email: vats7226@126.com; Ru-Lin Qian, MD. Department of Thoracic Surgery, Henan Chest Hospital, Zhengzhou 450000, China. Email: 13633860396@163.com.

Background: This cohort study aimed to compare the performance of the 2015 diagnostic criteria for malnutrition of the European Society of Clinical Nutrition and Metabolism (ESPEN), the Nutritional Risk Screening 2002 (NRS 2002), Malnutrition Universal Screening Tool (MUST), and Short-Form of MiniNutritional Assessment (MNA-SF) in detecting malnutrition risk and predicting postoperative complications and the failure of early oral feeding (EOF) programs in esophageal cancer patients.

Methods: The 4 tools were used to conduct malnutrition assessments before surgery. The patients were divided into the groups of severe malnutrition and mild/moderate malnutrition and the incidences of the endpoints were observed. Multivariable logistic regression and receiver operating characteristic (ROC) curve analyses were conducted.

Results: Two hundred and nineteen consecutive esophageal cancer patients were included in the study. The prevalence rates of severe malnutrition as determined by the ESPEN 2015 criteria, MUST, NRS 2002, and MNA-SF were 24.7\%, 29.7\%, 23.7\%, and 16.0\%, respectively. The moderate/severe malnutrition risk screened by the MUST had a high sensitivity (100.0\%) with malnutrition identified by the ESPEN 2015 criteria. In total, 42 (19.2\%) patients experienced major complications, and the incidence rate of EOF failure was $7.3 \%$. The severe malnutrition identified by the ESPEN 2015 criteria, MUST, and NRS 2002 were comparable in predicting the incidence of postoperative pulmonary complications, anastomotic leakage, readmission to intensive care units (ICUs), and EOF failure, but the ESPEN 2015 criteria was better in predicting postoperative overall complications, major complications, and delayed hospital discharge.

Conclusions: The ESPEN 2015 criteria specializes in identifying severe malnutrition and is better in predicting adverse surgical outcomes; however, the MUST and NRS 2002 are better superior in detecting early malnutrition and are also valuable in the perioperative management in esophageal surgery. It is recommended that the MUST be used as the malnutrition screening tool before the ESPEN 2015 criteria is applied.

Keywords: Early oral feeding (EOF); esophageal neoplasm; esophagectomy; postoperative complications; malnutrition 
Submitted Nov 05, 2021. Accepted for publication Dec 23, 2021.

doi: 10.21037/atm-21-6383

View this article at: https://dx.doi.org/10.21037/atm-21-6383

\section{Introduction}

Esophageal cancer is the 12th most common cancer and the 7 th most common cause of mortality worldwide (1). Patients with esophageal cancer naturally suffer from a nutritional risk because of metabolic effects and eating problems (2). Malnutrition has been widely confirmed to be associated with increased morbidity and mortality, prolonged hospitalization, and increased healthcare costs $(2,3)$. Malnourished patients undergoing esophagectomy can benefit from preoperative nutritional risk screening, systematic nutritional support and intensive perioperative management (3); however, a major obstacle to improve perioperative nutrition managements is the lack of standard criteria for diagnosing malnutrition.

Some objective nutritional screening tools, including the Malnutrition Universal Screening Tool (MUST) (4), Nutritional Risk Screening 2002 (NRS 2002) (5), and Short-Form of Mini-Nutritional Assessment (MNA-SF) (6), have been validated for nutritional risk screening, but few studies have compared the value of these tools in predicting adverse therapeutic outcomes after esophagectomy. Additionally, while the 2015 consensus on malnutrition diagnosis of the European Society of Clinical Nutrition and Metabolism (ESPEN) (7) which emphasizes lean body mass loss, has been validated in several medical fields $(8,9)$, its diagnostic efficiency for esophageal cancer with respect to clinical outcomes remains unknown.

Fast-track surgery has been increasingly introduced to esophageal cancer patients undergoing esophagectomy $(10,11)$. As a practice of fast-track surgery, the "non-tube no fasting" early oral feeding (EOF) program is the standard recovery model routinely used in our department after minimally invasive McKeown esophagectomy (McKeownMIE) and has shown to be effective at promoting bowel function, reducing inflammation reactions, and improving short-term quality of life (12-15). However, some patients must delay or discontinue EOF because of the occurrence of critical complications, severe abdominal distension, or poor oral intake $(12,14)$. We hypothesized that malnutrition, accompanied by compromised recovery potential and elevated complications risk, could have a negative effect on the implementation of the EOF program.
In this study, we sought to investigate the efficacy of the ESPEN 2015 criteria in screening malnutrition risk in esophageal cancer patients to the MUST, NRS 2002, and MNA-SF and the efficacy of these 4 tools in predicting the incidences of complications and EOF failure after esophagectomy.

We present the following article in accordance with the STROBE reporting checklist (available at https://dx.doi. org/10.21037/atm-21-6383).

\section{Methods}

\section{Study design and patients}

Detailed data of consecutive esophageal cancer patients from January 2018 to May 2019 at the Department of Thoracic Surgery, The Affiliated Cancer Hospital of Zhengzhou University were prospectively collected and retrospectively analyzed in this study. Malnutrition assessments using the ESPEN 2015 criteria, MUST, NRS 2002, and MNA-SF were conducted for all patients before surgery. To be eligible for inclusion in this study, patients had to meet the following inclusion criteria: (I) be aged from 20 to 80 years; (II) have a diagnosis of thoracic esophageal squamous cell carcinoma; (III) have undergone a McKeown-MIE; and (IV) have provided written informed consent. Patients were excluded from the study if they met any of the following exclusion criteria: (I) had an advanced tumor stage without surgical indications; (II) had impaired renal, hepatic or cardiovascular function; (III) had a history of previous gastrointestinal malignancies or gastrointestinal surgery; and/or (IV) had not undergone malnutrition assessments using any of the 4 tools. Patients were divided into severe and mild/moderate malnutrition groups according to the 4 tools to observe the incidences of the postoperative endpoints. The study was conducted in accordance with the Declaration of Helsinki (as revised in 2013). The study was approved by The Affiliated Cancer Hospital of Zhengzhou University Ethics Committee (No. 2018139). The requirement of informed consent was waived by The Affiliated Cancer Hospital of Zhengzhou University Ethics Committee due to the retrospective study design and minimal risk in research. 


\section{Data collection}

The parameters of body composition were assessed at 7:00 a.m. within 1 week before the operation by a bioelectrical impedance analysis (BCA-IB, Tsinghua Tongfang Co., Ltd., Beijing, China). Body mass index (BMI), fat-free mass, skeletal muscle mass, and fat mass were automatically measured. The fat-free mass index (FFMI) is calculated as fat-free mass in kilograms divided by height in square meters $\left(\mathrm{kg} / \mathrm{m}^{2}\right)$. Each patient's physical status was assessed according to the American Society of Anesthesiologists physical status (ASA-PS) classification and the Eastern Cooperative Oncology Group performance status (ECOG-PS) (15). Pulmonary function parameters and biochemical indexes were assessed within 1 week before the operation. Postoperative complications were defined and recorded according to the international consensus on the standardization of data collection for complications associated with esophagectomy and were graded according to the Clavien-Dindo classification of surgical complication. All patients were followed up once a week during the first 2 months and once a month during the first year after surgery.

\section{Malnutrition screening tools}

The contents and malnutrition screening approaches of the 4 tools are shown in Table S1. The ESPEN 2015 criteria is a group consensus proposed by the ESPEN and includes a decreased FFMI and BMI and weight loss history as evaluation elements (7). The MUST was devised for application in all adult patients across all healthcare settings using evidence-based criteria and has three parameters: BMI, weight loss, and acute disease (4). The NRS 2002 was derived from an analysis of controlled clinical trials and has its measurement parameters include weight loss, food intake, $\mathrm{BMI}$, and disease severity (5). The MNA-SF is a nutritional screening tool that was especially designed for elderly patients and aims to initially identify nutritional risks (6). Notably, the ESPEN 2015 criteria diagnoses whether or not patients are malnourished, while the other 3 tools aim to classify the risk malnutrition of as mild, moderate, or severe.

\section{Treatment strategy}

Patients were staged before and after surgery according to the eighth edition of the American Joint Committee on Cancer and the International Union Against Cancer staging manuals. Neoadjuvant chemotherapy followed by esophagectomy was the preferred strategy for treating patients with nodal disease spread $\left(\mathrm{cN}_{+}\right)$or transmural tumor invasion $(\mathrm{cT} \geq 3$ ) and ECOG-PS $\leq 2$. Patients identified as being nourished by the NRS 2002 were placed on an enteral nutrition support program from the early preoperative stage (7-10 days) under the guidance of dietitians; however, no patient was recommended for surgery until the nutritional status recovered to normal. Two experienced surgeons, each performing $>100$ McKeown-MIE procedures per year, performed the surgical procedures. All patients underwent McKeown-MIE with 2- or 3-field lymph node dissection, and a hand-sewn, 3 -layer embedded cervical esophagogastric anastomosis was constructed (12-15). No nasogastric tubes were routinely placed before or during surgery.

\section{EOF program}

We performed feasibility assessment for all patients before the administration of the EOF program on postoperative day 1 (POD 1). The exclusion criteria for the EOF program included the occurrence of emergency intraoperative events, the appearance of acute organ dysfunction, and defined vocal cord palsy based on a bedside endoscopic examination $(12,14)$. Patients ineligible for the EOF program were introduced to a late-oral-feeding protocol whereby nasogastric and nasoenteral feeding tubes were placed with the help of interventional radiology on POD 1, and enteral and parenteral nutrition was provided (12).

Patients eligible for the EOF program were encouraged to start oral feeding on POD 1 in accordance with the "chew 50 times per bite" principle under the observation and guidance of at least 1 clinician and 1 dietician (14). There were no strict limitations on the types of solid foods and nutrition times, which were based on the patient's wishes. Patients were also allowed 6-8 eating episodes per day according to their desire; however, they were advised to avoid feeling full and the feeling of distension. Patients' caloric requirements were calculated by a nutritionist based on the modified Harris-Benedict formula $+30 \%$ for postoperative energy requirements (16). The oral intake of calories was calculated within 3 days after surgery, and additional intravenous nutrition was supplemented according to the postoperative energy requirements. Intravenous fluid infusion was routinely stopped on POD 4 or continued according to patients' oral intake and illness conditions. 


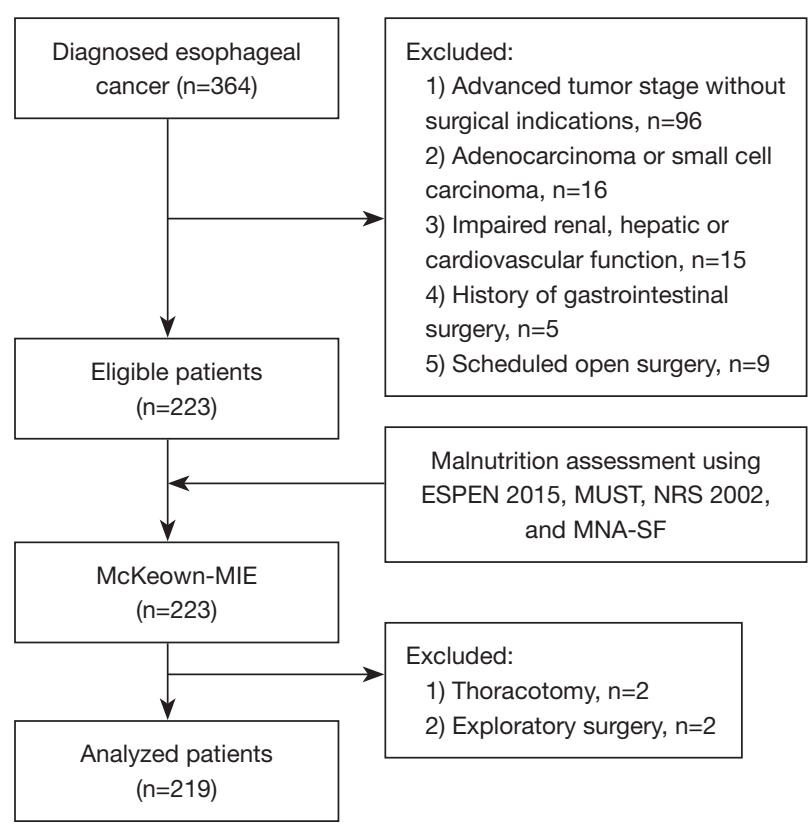

Figure 1 Flowchart of study participants. McKeown-MIE, minimally invasive McKeown esophagectomy; ESPEN 2015, European Society of Clinical Nutrition and Metabolism consensus on malnutrition diagnosis [2015]; MUST, Malnutrition Universal Screening Tool; NRS 2002, Nutritional Risk Screening 2002; MNA-SF, Short-Form of Mini-Nutritional Assessment.

\section{Endpoints}

The primary endpoint was the performance of the global leadership initiative on malnutrition criteria in screening malnutrition risk compared to the MUST, NRS 2002, and MNA-SF. The second point was the value of the malnutrition identified by the 4 tools in predicting postoperative major complications and EOF failure. Major complications were defined as being $\geq$ Clavien-Dindo grade III. EOF failure was defined as a pause in oral feeding for $>2$ days because of the occurrence of postoperative emergency events or the need for intravenous nutrition for $>6$ days because of poor oral intake. We also focused on other adverse surgery-related outcomes, including specific complications, readmission to intensive care units (ICUs), and delayed hospital discharge.

\section{Statistical analysis}

To investigate the characteristics of malnourished patients, an analysis of variance, Mann-Whitney U test, Pearson' $\chi^{2}$ test, Fisher's exact test, and Kruskal-Wallis test were used according to the characteristics of the data. Cohen's $\mathrm{K}$ statistic and a receiver operating characteristic (ROC) curve analysis were used to determine the diagnostic concordance of malnutrition risk between the ESPEN 2015 criteria and the MUST, NRE 2002, and MNA-SF. The sensitivity and specificity values of the MUST, NRS 2002, and MNA-SF compared to the ESPEN 2015 criteria for malnutrition screening were also calculated. Multivariable logistic regression and ROC curve analyses were used to assess the predictive value of these 4 tools in relation to the endpoints (17). The continuous variables were grouped according to the median, quartiles, and practical significance to be included in multivariable analyses. A two-tailed $\mathrm{P}$ value $<0.05$ was considered statistically significant when exploring the data characteristics. A two-tailed adjusted $\mathrm{P}$ value $<0.05 / 6$ (i.e., $<0.008$ ) was considered statistically significant when comparing the endpoints among the 4 tools, according to the Bonferroni correction. All analyses were conducted using IBM SPSS Statistics for Windows (version 22.0, IBM Corp., Armonk, NY, USA).

\section{Results}

During the study period, a total of 364 esophageal cancer patients were diagnosed at our department (see Figure 1). Following the study criteria, 223 eligible patients were identified, and 219 patients were ultimately included in the study after excluding cases of thoracotomy and exploratory surgery. All the included patients were followed up for at least 3 months, except for $2(0.9 \%)$ patients who died due to critical complications within 28 days of the surgery.

\section{Nutritional assessment}

The cross-tabulation presenting the details of malnutrition risk identified by the ESPEN 2015 criteria and MUST, NRS 2002, and MNA-SF are shown in Table S2. The prevalence rates of severe malnutrition as recognized by the ESPEN 2015 criteria, MUST, NRS 2002, and MNA-SF were $27.4 \%, 29.7 \%, 23.7 \%$, and $16.0 \%$, respectively (see Figure 2). As Table 1 (evaluation 1) shows, all the moderate/ severe malnutrition risk screenings by the MUST, NRS 2002, and MNA-SF showed a high sensitivity (100.0\%, $96.3 \%$, and $96.3 \%$, respectively) with malnutrition as identified by the ESPEN 2015 criteria, but with poor specificity $(47.9 \%, 41.2 \%$, and $29.1 \%$, respectively). In relation to the detection of severe malnutrition (see Table 1, evaluation 2), the MUST showed the highest sensitivity 


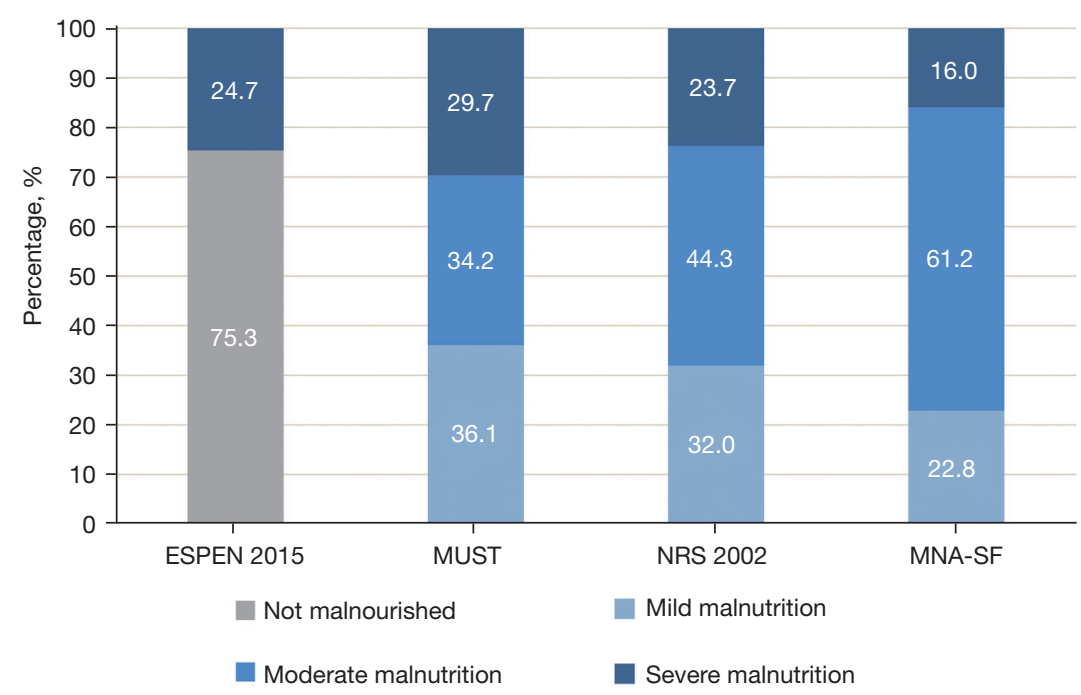

Figure 2 Distribution of malnutrition risk screened by the 4 tools. The ESPEN 2015 diagnoses whether or not patients are malnourished, while the other 3 tools classify the severity of the malnutrition as mild, moderate, or severe. ESPEN 2015, European Society of Clinical Nutrition and Metabolism consensus on malnutrition diagnosis [2015]; MUST, Malnutrition Universal Screening Tool; NRS 2002, Nutritional Risk Screening 2002; MNA-SF, Short-Form of Mini-Nutritional Assessment.

Table 1 The malnutrition screening property of the MUST, NRS 2002, and MNA-SF compared to the ESPEN 2015 criteria

\begin{tabular}{|c|c|c|c|c|c|c|}
\hline Parameters & \multicolumn{3}{|c|}{ Evaluation $1^{a}$} & \multicolumn{3}{|c|}{ Evaluation $2^{b}$} \\
\hline Sensitivity (\%) & 100.0 & 96.3 & 96.3 & 75.9 & 59.3 & 53.7 \\
\hline Specificity (\%) & 47.9 & 41.2 & 29.1 & 85.5 & 87.9 & 96.4 \\
\hline Positive predictive value (\%) & 38.6 & 34.9 & 30.8 & 63.1 & 61.5 & 82.9 \\
\hline Positive likelihood ratio & 1.92 & 1.64 & 1.36 & 5.22 & 4.89 & 14.77 \\
\hline Negative likelihood ratio & 0 & 0.09 & 0.13 & 0.28 & 0.46 & 0.48 \\
\hline$K$ value $(P)^{c}$ & $0.312(\mathrm{P}<0.001)$ & $0.236(\mathrm{P}<0.001)$ & $0.148(\mathrm{P}<0.001)$ & $0.574(\mathrm{P}<0.001)$ & $0.477(\mathrm{P}<0.001)$ & $0.568(\mathrm{P}<0.001)$ \\
\hline AUC (95\% Cl) & $\begin{array}{c}0.739 \\
(0.675-0.803)\end{array}$ & $\begin{array}{c}0.688 \\
(0.616-0759)\end{array}$ & $\begin{array}{c}0.627 \\
(0.549-0.705)\end{array}$ & $\begin{array}{c}0.807 \\
(0.733-0.880)\end{array}$ & $\begin{array}{c}0.736 \\
(0.651-0.821)\end{array}$ & $\begin{array}{c}0.750 \\
(0.663-0.838)\end{array}$ \\
\hline
\end{tabular}

\footnotetext{
${ }^{a}$, evaluation of moderate/severe malnutrition screening property of the other tools compared to the ESPEN 2015 criteria; ${ }^{b}$, evaluation of severe malnutrition screening property of the other tools compared to the ESPEN 2015 criteria; ' $\mathrm{K}$ value derived from Cohen's $\mathrm{K}$ statistics, reflecting the consistency of qualitative variables: $\mathrm{K}<0.400$ poor agreement; $\mathrm{K}=0.400-0.750$ fair-good; $\mathrm{K}>0.750$ excellent agreement beyond chance. MUST, Malnutrition Universal Screening Tool; NRS 2002, Nutritional Risk Screening 2002; MNA-SF, Short-Form of Mini-Nutritional Assessment; ESPEN 2015, European Society of Clinical Nutrition and Metabolism consensus on malnutrition diagnosis [2015]; AUC, area under the receiver operating characteristic curve; $\mathrm{Cl}$, confidence interval.
}

(75.9\%), negative predictive value $(91.6 \%)$ and had good consistency $[\mathrm{K}=0.574$, area under the ROC curve (AUC) $=0.807]$ with the ESPEN 2015 criteria, while the MNASF showed the highest specificity $(96.4 \%)$ and positive predictive value (82.9\%) with the ESPEN 2015 criteria.

\section{Malnutrition and clinicopathological characteristics}

The patients' clinicopathological characteristics are presented in Table 2. Compared to patients with mild/ moderate malnutrition, those with severe malnutrition as 


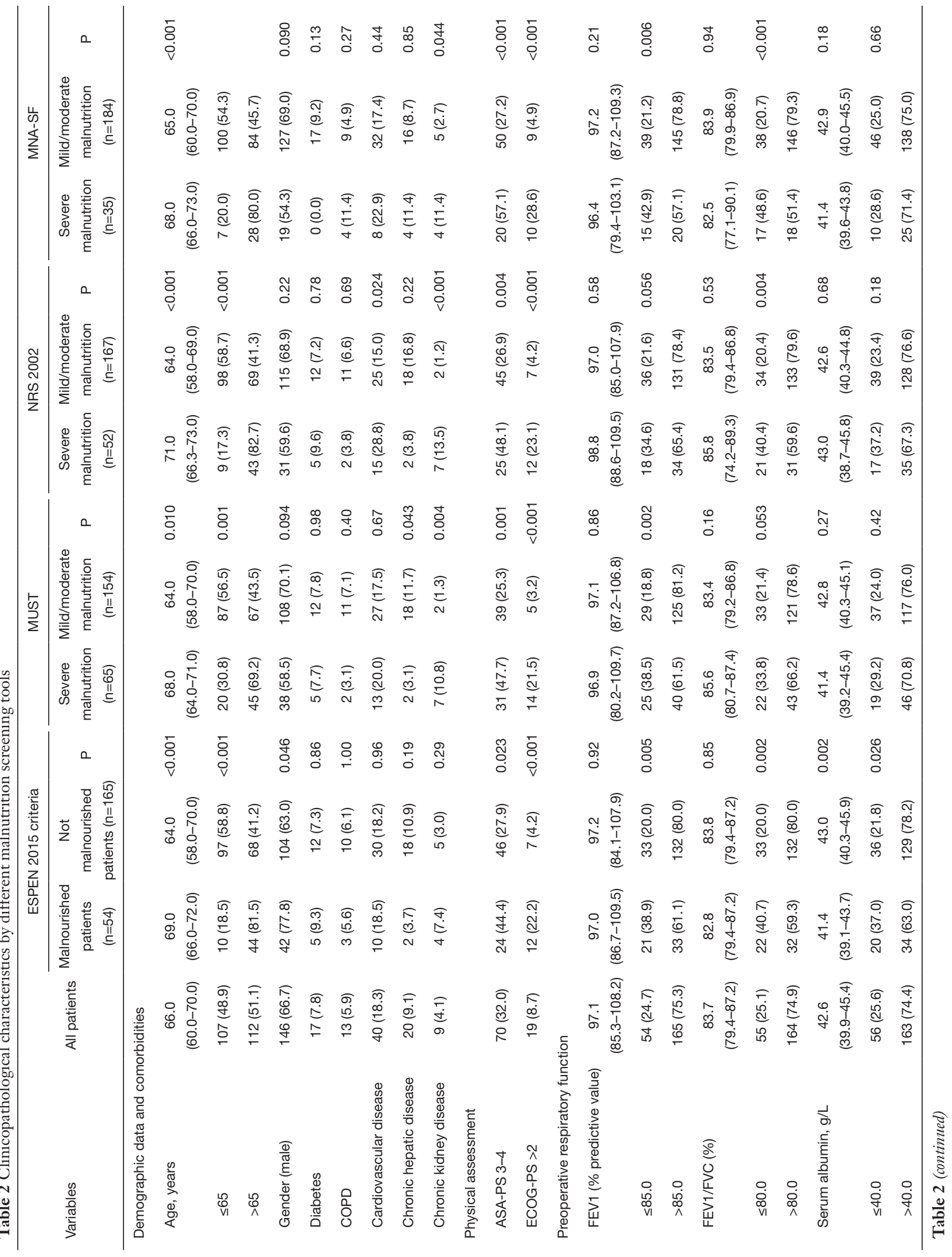




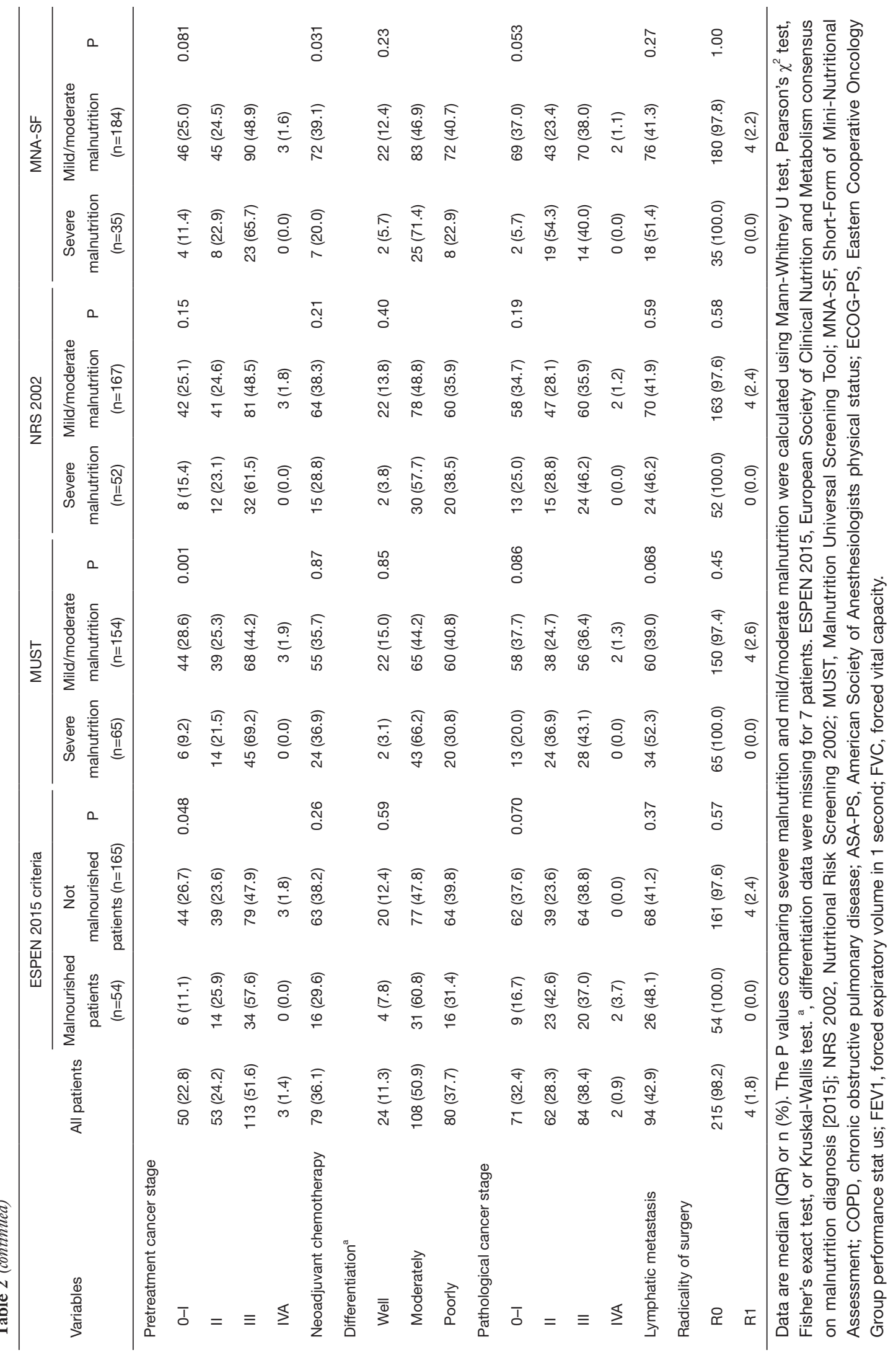


identified by the ESPEN 2015 criteria, MUST, NRS 2002, and MNA-SF shared the following common characteristics: an advanced age, a higher ASA-PS 3-4, and a higher ECOG-PS score (all $\mathrm{P}<0.05$ ). Compromised respiratory function [i.e., reduced forced expiratory volume in 1 second (FEV1) (\% predictive value) and reduced FEV1/forced vital capacity (FVC)], was observed in the malnourished patients identified by the ESPEN 2015 criteria and MNA-SF (all $\mathrm{P}<0.05)$. Notably, malnutrition identified by the ESPEN 2015 criteria was found to be significantly associated with lower levels of serum albumin $(\mathrm{P}=0.002)$, but this was not observed in other groups. Additionally, malnourished patients diagnosed by the ESPEN 2015 criteria had a more advanced clinical cancer stage $(\mathrm{P}=0.048)$, but there was no significant difference in the administration of neoadjuvant chemotherapy, tumor differentiation, and pathological cancer stage between the malnourished and the nonmalnourished patients.

\section{Malnutrition and perioperative parameters}

According to the univariable analyses (see Table S3), severely malnourished patients as identified by the 4 tools had increased incidence rates of pneumonia, respiratory failure, infectious complications, overall complications, and readmission to ICU (all $\mathrm{P}<0.05$ ). Severe malnutrition ass identified by the ESPEN 2015 criteria, MUST, and NRS 2002 was found to be significantly associated with the incidence of anastomotic leakage (all $\mathrm{P}<0.05)$. In total, $42(19.2 \%)$ patients experienced major complications after surgery. The severity grades of complications in patients with severe malnutrition risk were significantly higher than those in patients with mild/moderate malnutrition risk (all $\mathrm{P}<0.05)$. A total of $199(90.8 \%)$ patients participated in the EOF program after the feasibility assessment, but 16 (7.3\%) patients failed to complete the EOF program, with the direct reasons being the occurrence of critical complications (9 patients), serious stomach distention (2 patients), and poor oral intake (5 patients). The median postoperative hospital stay of the 219 patients was $9.0(8.0-12.0)$ days, and delayed hospital discharge was defined as a postoperative stay $>12$ days.

\section{Value of the 4 malnutritional screening tools for predicting endpoints}

In relation to the multivariable logistical analyses (see Table 3), after adjusting for the cofounding factors of clinicopathological parameters and intraoperative parameters, severe malnutrition as identified by the 4 tools was commonly associated with the occurrence of respiratory complications and EOF failure (all $\mathrm{P}<0.008)$. Severe malnutrition as identified by the ESPEN 2015 criteria, MUST and NRS 2002 performed well in predicting the occurrence of major complications and readmission to ICU (all $\mathrm{P}<0.008$ ). Malnutrition diagnosed by ESPEN 2015 criteria was also predictive of postoperative anastomotic leakage [odds ratio $(\mathrm{OR})=17.72 ; \mathrm{P}=0.008$ ], infectious complications $(\mathrm{OR}=6.22 ; \mathrm{P}=0.002)$, overall complications $(\mathrm{OR}=15.48 ; \mathrm{P}<0.001)$ and delayed hospital discharge (OR $=4.83 ; \mathrm{P}<0.001)$, but the first value was not statistically significant after the Bonferroni correction.

The predictive value of the 4 nutritional screening tools for endpoints according to AUC is shown in Figure 3. The ESPEN 2015 criteria had the highest AUCs for predicting the incidence of postoperative respiratory complications [AUC $=0.777 ; 95 \%$ confidence interval (CI): 0.689-0.866; see Figure 3A], major complications (AUC $=0.730 ; 95 \% \mathrm{CI}$ : $0.637-0.823$; see Figure $3 E$ ), and delayed hospital discharge (AUC $=0.702 ; 95 \%$ CI: 0.611-0.793; see Figure 3H). The ESPEN 2015 criteria, MUST and NRS 2002 all showed moderate efficacy in predicting the incidence of anastomotic leakage (see Figure 3B), readmission to ICU (see Figure 3F), and EOF failure (see Figure $3 G$ ). However, the MNA-SF performed poorly in predicting the incidence of most of the endpoints.

\section{Discussion}

This study compared the efficacy of the ESPEN 2015 criteria in screening malnutrition risk and predicting adverse surgery-related outcomes in esophageal cancer patients to the efficacy of the MUST, NRS 2002, and MNA-SF. Our analyses indicate that ESPEN 2015 criteria performed well at diagnosing severe malnourishment, but lacked sensitivity in detecting early malnutrition risk. All the parameters included in the ESPEN 2015 criteria (i.e., low BMI, weight loss, and reduced FFMI), are direct consequences of nutritional deficiency and body composition loss (7). Thus, the ESPEN 2015 criteria provides a precise method for identifying severe metabolic risk, and consequently identifies fewer patients as having a malnutrition risk, which enhances its relevance in determining diminished physical performance, and accounts for its good efficacy in predicting adverse therapeutic outcomes. Specifically, a decreased FFMI is a crucial criterion among the ESPEN 


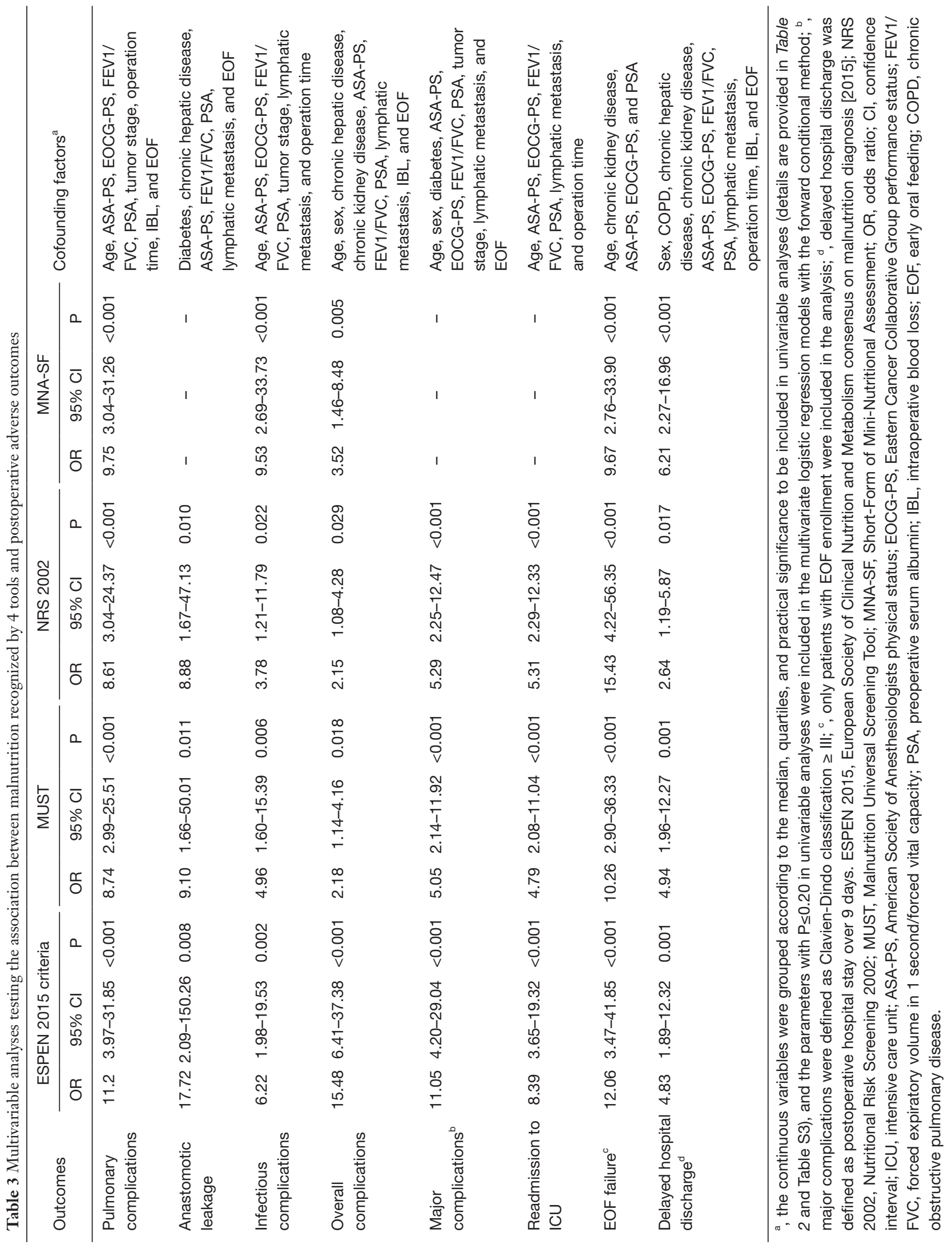



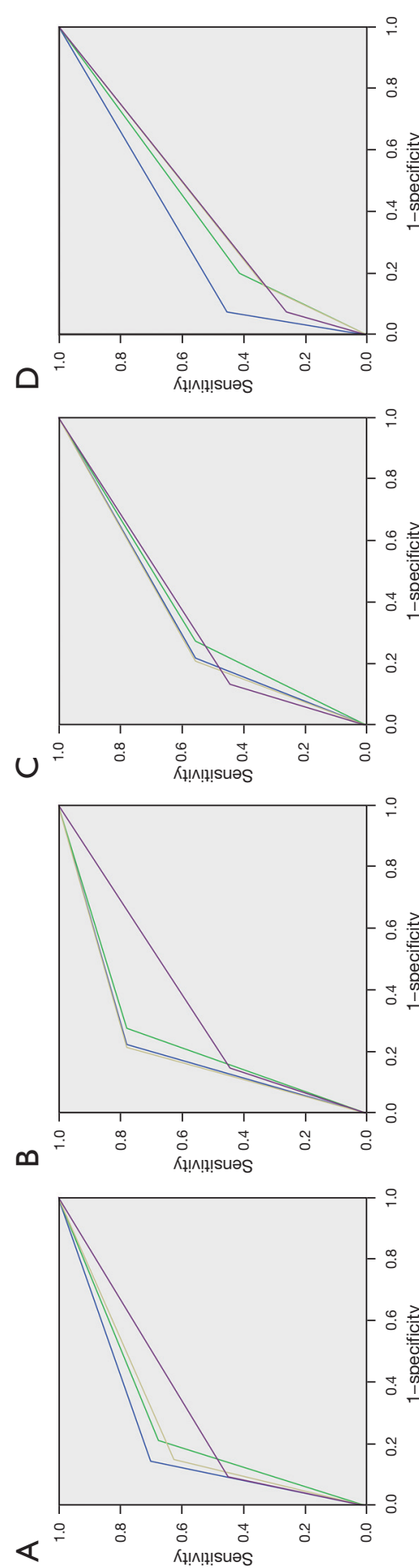
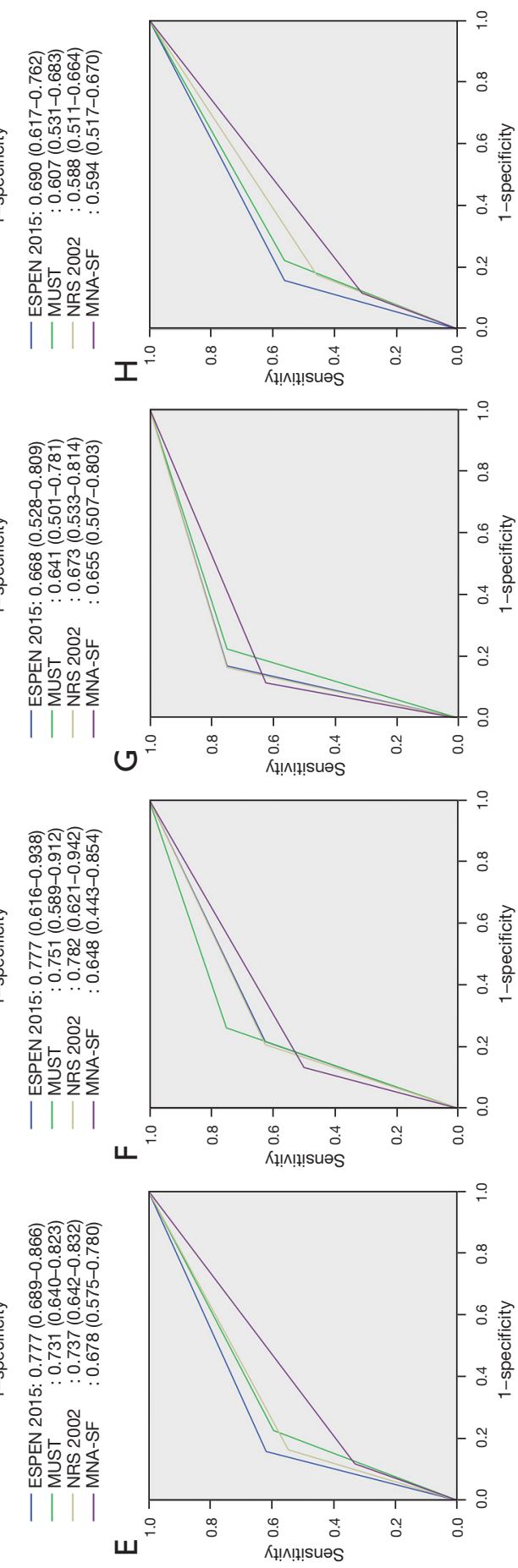

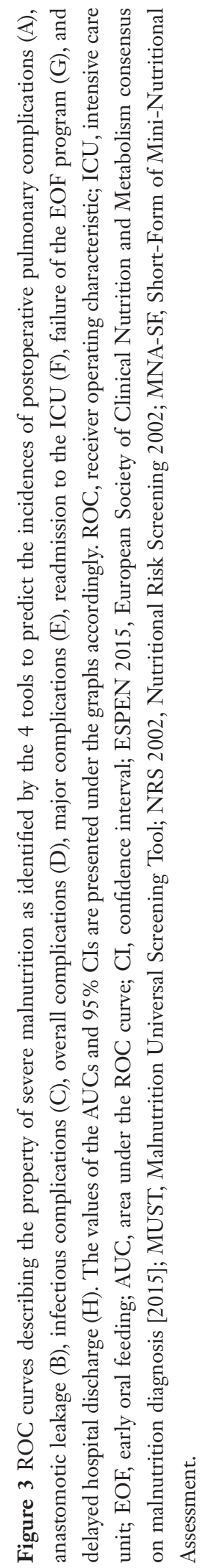


2015 criteria and has been reported to be associated with an increased risk of mortality in hospitalized patients (9). A reduced FFMI is particularly associated with skeletal muscle depletion and the incidence of sarcopenia and cachexia, which are associated with adverse therapeutic outcomes (18).

To determine malnutrition, a series of parameters and assessment tools have been proposed and validated in medical practice (17). A prospective study of 608 patients who underwent gastrointestinal surgery confirmed the value of the NRS 2002 in predicting the severity of postoperative complications (19). High-malnutrition risk as identified by MUST has also been reported to be associated with the incidence of complications in gastrointestinal surgery (20). The clinical performance of the MNA-SF in gastrointestinal surgery has been insufficiently reported and was proven to be poor this time. Our study first showed the superiority of the ESPEN 2015 criteria in predicting postoperative complications and EOF failure in esophageal cancer patients, which were independent of the clinicopathological characteristics and intraoperative parameters. Notably, malnutrition as identified by the ESPEN 2015 criteria is accompanied by compromised respiratory function and decreased serum albumin levels, which may be related to the loss of fat-free mass, especially reduced skeletal muscle mass, which indicates the superiority of the ESPEN 2015 criteria in detecting deteriorated body conditions (18). Apart from nutrition assessment tools included in this study, the Global Leadership Initiative on Malnutrition have recently established global diagnostic criteria and classification methods for malnutrition; these criteria showed good performance in diagnosing and classifying malnutrition in people with esophageal cancer undergoing esophagectomy (21). Nutritional risk indexes based on the examination of biochemical and clinical indexes, such as the Geriatric Nutritional Risk Index, were reported to be valuable in simplifying nutrition assessment and predicting therapeutic outcomes in esophageal cancer patients (22).

EOF successfully reduces the postoperative stress caused by fasting and had advantages in accelerating postoperative recovery $(12,13)$; however, we do have concerns about patients who experience EOF failure after EOF enrollment. The occurrence of EOF failure not only interferes with the recovery of digestive function, but may also aggravate the physical and mental stress of patients. As results showed, severe malnutrition-related complications, such as anastomotic leakage and respiratory failure, are the main factors that prevent patients from oral feeding; thus, the preoperative detection and management of severe malnutrition could be a valuable way of promoting EOF program. Additionally, the poor oral intake of malnourished patients is related to the depletion of fat-free mass, especially the degeneration of the oropharyngeal muscle; thus, the preoperative exercise of oropharyngeal motility may also promote the implementation of the EOF program (23).

Nutritional assessments and interventions are important in gastrointestinal cancers given the significant malnutrition risk. Clinical decision makers recommend adopting appropriate malnutrition screening tools according to their purposes and resources. The ESPEN 2015 criteria showed superiority in predicting adverse surgery-related outcomes, but requires body composition analyses and lacks a capability to detect early malnutrition risk. The MUST and NRS 2002 are not only convenient but also valuable for medical departments with limited medical resources. Notably, give the good sensitivity of the MUST and the ESPEN 2015 criteria in detecting moderate/ severe malnutrition risk, the MUST could serve as the primary malnutrition assessment before the application of the ESPEN 2015 criteria to economize medical resources. Additionally, multimodal cancer prehabilitation programs are recommended to promote the therapeutic benefits of esophageal surgery (24). Notably, nutritional support and physical exercise are prerequisites for rebuilding peripheral protein mass and improving surgical outcomes (25), exercise of the respiratory muscles promotes respiratory function and reduces pulmonary complications (26), and the preoperative reversal of low serum albumin levels should promote incision healing and reduce the incidence of anastomotic complications (27).

Our analysis had certain limitations that were mainly related to its nature a single-center study. Only patients who underwent McKeown-MIE with hand-sewn cervical anastomosis were included in this study; thus, the predictive values of the 4 tools in other surgical procedures and anastomosis techniques requires further investigation. Only a small sample of Asian patients, with the main tumor histology type of squamous cell, over a short period were enrolled in the study. Patients' nutritional data before the neoadjuvant chemotherapy was unavailable. The small percentage of neoadjuvant chemotherapy may compromise the applicability of our data analysis. Future interventional studies need to be conducted to investigate the benefits of nutritional interventions based on the use of the ESPEN 2015 criteria, NRS 2002, MUST and MNA-SF.

In conclusion, the ESPEN 2015 criteria specialize in detecting severe malnutrition and was better in predicting 
adverse surgical outcomes in esophageal cancer patients than the MUST, NRS 2002, and MNA-SF. However, the MUST and NRS 2002 are also valuable in predicting postoperative outcomes and possess superiority in detecting early malnutrition risk. Notably, it is recommended that the MUST be used to conduct the primary malnutrition assessment before the ESPEN 2015 criteria are applied. Multimodal prehabilitation programs are recommended for malnutrition patients to reduce the risk of complications and promote the implementation of the EOF program.

\section{Acknowledgments}

Funding: This work was funded by the Medical and Health Technology Innovation Project of Chinese Academy of Medical Sciences (grant No. 2018-12M-3-003) (SpatialTemporal Mapping Analysis on Chinese Cancer Burden).

\section{Footnote}

Reporting Checklist: The authors have completed the STROBE reporting checklist. Available at https://dx.doi. org/10.21037/atm-21-6383

Data Sharing Statement: Available at https://dx.doi. org/10.21037/atm-21-6383

Conflicts of Interest: All authors have completed the ICMJE uniform disclosure form (available at https://dx.doi. org/10.21037/atm-21-6383). The authors have no conflicts of interest to declare.

Ethical Statement: The authors are accountable for all aspects of the work in ensuring that questions related to the accuracy or integrity of any part of the work are appropriately investigated and resolved. The study was conducted in accordance with the Declaration of Helsinki (as revised in 2013). The study was approved by The Affiliated Cancer Hospital of Zhengzhou University Ethics Committee (No. 2018139). The requirement of informed consent was waived by The Affiliated Cancer Hospital of Zhengzhou University Ethics Committee due to the retrospective study design and minimal risk in research

Open Access Statement: This is an Open Access article distributed in accordance with the Creative Commons Attribution-NonCommercial-NoDerivs 4.0 International License (CC BY-NC-ND 4.0), which permits the non- commercial replication and distribution of the article with the strict proviso that no changes or edits are made and the original work is properly cited (including links to both the formal publication through the relevant DOI and the license). See: https://creativecommons.org/licenses/by-nc-nd/4.0/.

\section{References}

1. Global Burden of Disease Cancer Collaboration; Fitzmaurice C, Abate D, et al. Global, Regional, and National Cancer Incidence, Mortality, Years of Life Lost, Years Lived With Disability, and Disability-Adjusted LifeYears for 29 Cancer Groups, 1990 to 2017: A Systematic Analysis for the Global Burden of Disease Study. JAMA Oncol 2019;5:1749-68.

2. Weimann A, Braga M, Carli F, et al. ESPEN guideline: Clinical nutrition in surgery. Clin Nutr 2017;36:623-50.

3. Chen MJ, Wu IC, Chen YJ, et al. Nutrition therapy in esophageal cancer-Consensus statement of the Gastroenterological Society of Taiwan. Dis Esophagus 2018. doi: 10.1093/dote/doy016.

4. Stratton RJ, Hackston A, Longmore D, et al. Malnutrition in hospital outpatients and inpatients: prevalence, concurrent validity and ease of use of the 'malnutrition universal screening tool' ('MUST') for adults. Br J Nutr 2004;92:799-808.

5. Kondrup J, Rasmussen $\mathrm{HH}$, Hamberg $\mathrm{O}$, et al. Nutritional risk screening (NRS 2002): a new method based on an analysis of controlled clinical trials. Clin Nutr 2003;22:321-36.

6. Rubenstein LZ, Harker JO, Salvà A, et al. Screening for undernutrition in geriatric practice: developing the shortform mini-nutritional assessment (MNA-SF). J Gerontol A Biol Sci Med Sci 2001;56:M366-72.

7. Cederholm T, Bosaeus I, Barazzoni R, et al. Diagnostic criteria for malnutrition - An ESPEN Consensus Statement. Clin Nutr 2015;34:335-40.

8. Rondel ALMA, Langius JAE, de van der Schueren MAE, et al. The new ESPEN diagnostic criteria for malnutrition predict overall survival in hospitalised patients. Clin Nutr 2018;37:163-8.

9. Marco E, Sánchez-Rodríguez D, Dávalos-Yerovi VN, et al. Malnutrition according to ESPEN consensus predicts hospitalizations and long-term mortality in rehabilitation patients with stable chronic obstructive pulmonary disease. Clin Nutr 2019;38:2180-6.

10. Chen L, Sun L, Lang Y, et al. Fast-track surgery improves postoperative clinical recovery and cellular and humoral 
immunity after esophagectomy for esophageal cancer. BMC Cancer 2016;16:449.

11. Shewale JB, Correa AM, Baker CM, et al. Impact of a Fast-track Esophagectomy Protocol on Esophageal Cancer Patient Outcomes and Hospital Charges. Ann Surg 2015;261:1114-23.

12. Sun HB, Li Y, Liu XB, et al. Early Oral Feeding Following McKeown Minimally Invasive Esophagectomy: An Openlabel, Randomized, Controlled, Noninferiority Trial. Ann Surg 2018;267:435-42.

13. Sun HB, Li Y, Liu XB, et al. Impact of an Early Oral Feeding Protocol on Inflammatory Cytokine Changes After Esophagectomy. Ann Thorac Surg 2019;107:912-20.

14. Zhu Z, Li Y, Zheng Y, et al. Chewing 50 times per bite could help to resume oral feeding on the first postoperative day following minimally invasive oesophagectomy. Eur J Cardiothorac Surg 2018;53:325-30.

15. Wang P, Li Y, Sun H, et al. Analysis of the associated factors for severe weight loss after minimally invasive McKeown esophagectomy. Thorac Cancer 2019;10:209-18.

16. Weijs TJ, Berkelmans GH, Nieuwenhuijzen GA, et al. Immediate Postoperative Oral Nutrition Following Esophagectomy: A Multicenter Clinical Trial. Ann Thorac Surg 2016;102:1141-8.

17. Poulia KA, Yannakoulia M, Karageorgou D, et al. Evaluation of the efficacy of six nutritional screening tools to predict malnutrition in the elderly. Clin Nutr 2012;31:378-85.

18. Muscaritoli M, Anker SD, Argilés J, et al. Consensus definition of sarcopenia, cachexia and pre-cachexia: joint document elaborated by Special Interest Groups (SIG) "cachexia-anorexia in chronic wasting diseases" and "nutrition in geriatrics". Clin Nutr 2010;29:154-9.

19. Schiesser M, Müller S, Kirchhoff P, et al. Assessment of a novel screening score for nutritional risk in predicting complications in gastro-intestinal surgery. Clin Nutr

Cite this article as: Hua XH, Shi KF, Yu YK, Li HM, Ma F, Sun HB, Qian RL, Li Y. Nutritional assessment in esophageal fast-track surgery: comparisons of 4 objective malnutrition screening tools. Ann Transl Med 2022;10(1):20. doi: 10.21037/ atm-21-6383
2008;27:565-70.

20. van der Kroft G, Janssen-Heijnen MLG, van Berlo CLH, et al. Evaluation of nutritional status as an independent predictor of post-operative complications and morbidity after gastro-intestinal surgery. Clin Nutr ESPEN 2015;10:e129-33.

21. Wang P, Chen X, Liu Q, et al. Good performance of the Global Leadership Initiative on Malnutrition criteria for diagnosing and classifying malnutrition in people with esophageal cancer undergoing esophagectomy. Nutrition 2021;91-92:111420.

22. Wang PY, Chen XK, Liu Q, et al. Application of four nutritional risk indexes in perioperative management for esophageal cancer patients. J Cancer Res Clin Oncol 2021;147:3099-111.

23. Fearon K, Arends J, Baracos V. Understanding the mechanisms and treatment options in cancer cachexia. Nat Rev Clin Oncol 2013;10:90-9.

24. Silver JK, Baima J. Cancer prehabilitation: an opportunity to decrease treatment-related morbidity, increase cancer treatment options, and improve physical and psychological health outcomes. Am J Phys Med Rehabil 2013;92:715-27.

25. Gillis C, Buhler K, Bresee L, et al. Effects of Nutritional Prehabilitation, With and Without Exercise, on Outcomes of Patients Who Undergo Colorectal Surgery: A Systematic Review and Meta-analysis. Gastroenterology 2018;155:391-410.e4.

26. Agrelli TF, Borges MC, Cunha FMRD, et al. Combination of preoperative pulmonary and nutritional preparation for esophagectomy. Acta Cir Bras 2018;33:67-74.

27. Sathianathen NJ, Kwaan M, Lawrentschuk N, et al. Adverse impact of malnutrition markers on major abdominopelvic cancer surgery. ANZ J Surg 2019;89:509-14.

(English Language Editor: L. Huleatt) 


\section{Supplementary}

Table S1 Assessment indexes and approaches of malnutrition screening tools

\begin{tabular}{|c|c|c|c|c|c|}
\hline Tools & $\begin{array}{l}\text { Year of } \\
\text { validation }\end{array}$ & Characteristics & Applied parameters & \multicolumn{2}{|c|}{ Assessment approaches } \\
\hline ESPEN 2015 & 2015 & $\begin{array}{l}\text { Diagnosis consensus } \\
\text { of malnutrition } \\
\text { independent of clinical } \\
\text { setting and etiology }\end{array}$ & $\begin{array}{l}\text { Option 1: } \mathrm{BMl}<18.5 \mathrm{~kg} / \mathrm{m}^{2} \text {; option 2: } \\
\text { unintentional weight loss (mandatory) and at } \\
\text { least one of either reduced } \mathrm{BMI} \text { or low FFMI }\end{array}$ & $\begin{array}{l}\text { Either } 1 \text { or } 2 \\
\text { Neither } 1 \text { or } 2\end{array}$ & $\begin{array}{l}\text { Malnourished } \\
\text { Not malnourished }\end{array}$ \\
\hline NRS 2002 & 2002 & $\begin{array}{l}\text { Based on analyses } \\
\text { of controlled clinical } \\
\text { trials }\end{array}$ & $\begin{array}{l}\text { Weight loss, food intake, BMI, and severity } \\
\text { of disease }\end{array}$ & $\begin{array}{l}\text { Score } 0-2 \\
\text { Score } 3-4 \\
\text { Score } \geq 5\end{array}$ & $\begin{array}{l}\text { Mild risk } \\
\text { Moderate risk } \\
\text { Severe risk }\end{array}$ \\
\hline
\end{tabular}

ESPEN 2015, European Society of Clinical Nutrition and Metabolism consensus on malnutrition diagnosis [2015]; NRS 2002, Nutritional Risk Screening 2002; MUST, Malnutrition Universal Screening Tool; MNA-SF, Short-Form of Mini-Nutritional Assessment; BMI, body mass index; FFMI, fat-free mass index.

Table S2 Cross-tabulation of malnutrition risk screened by ESPEN 2015 and the other four tools

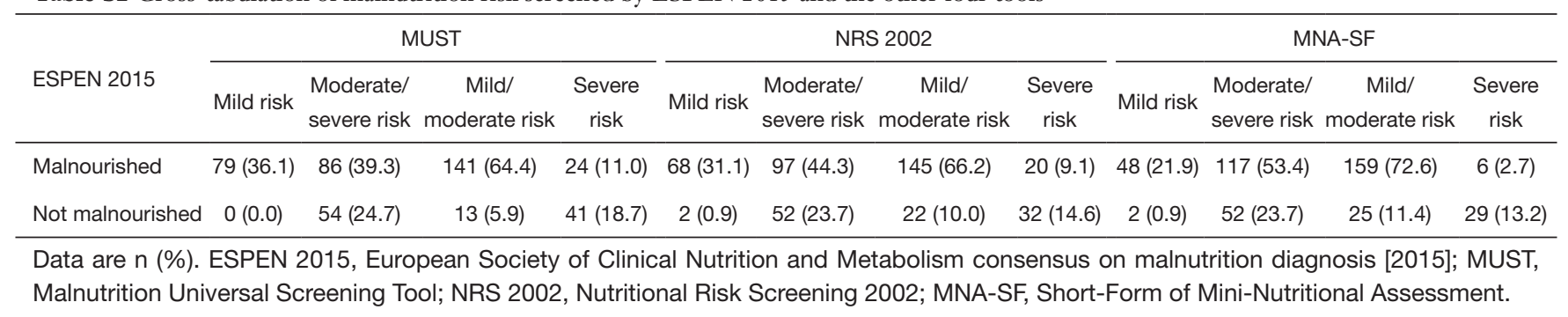




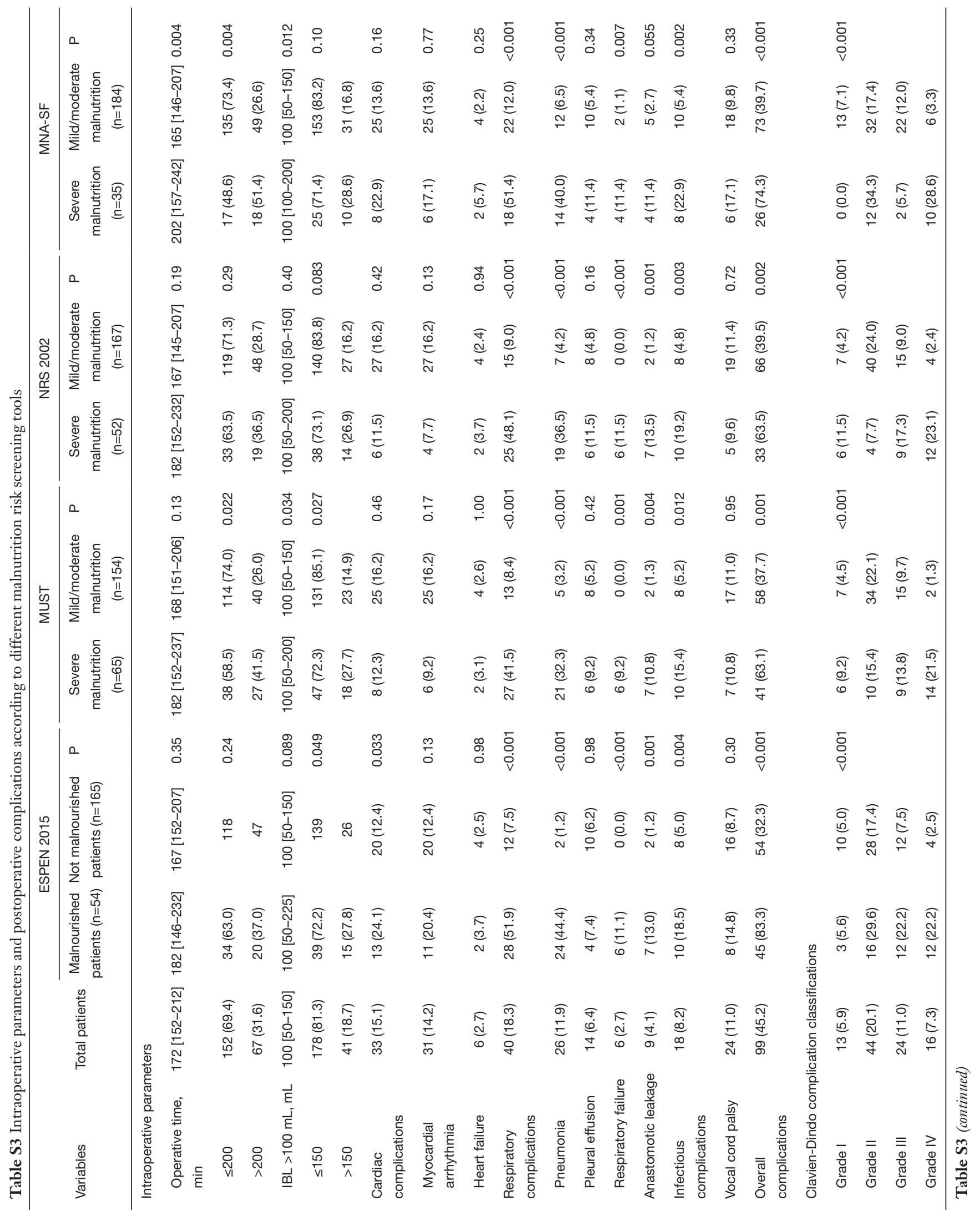




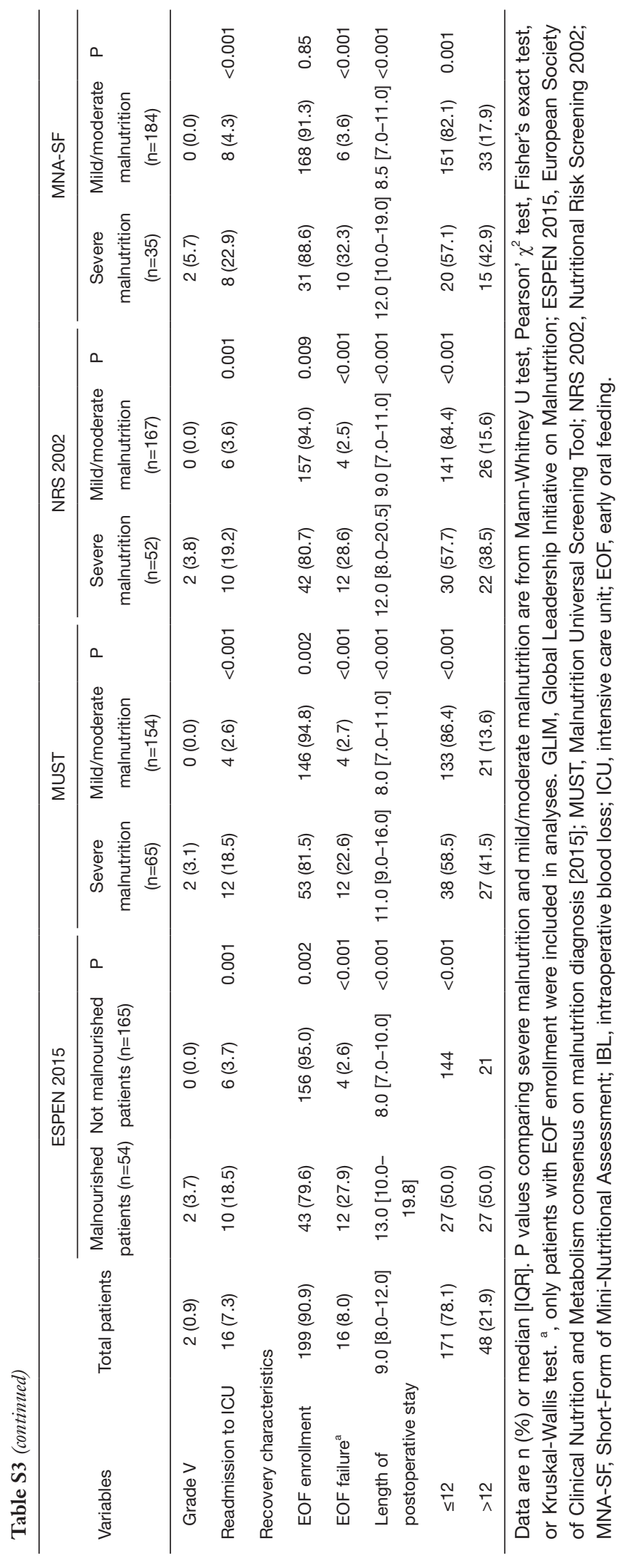

